\title{
Spontaneous recovery evoked by a nonreinforced stimulus
}

\author{
MARK D. HOLDER and KENNETH R. BURSTEIN \\ Simon Fraser University, Burnaby, British Columbia V5A 1S6, Canada
}

\begin{abstract}
Pigeons (nine groups of seven) were given one acquisition session of 20 trials. The following day their responding was extinguished, and after a 30-min rest period they were tested for residual response strength. The groups differed with respect to (1) whether a distinctive stimulus was presented during acquisition on the first trial, the 15th trial, or not at all, (2) whether or not responses to the distinctive stimulus were reinforced, and (3) whether a distinctive stimulus, a standard stimulus, or a completely novel stimulus was presented on the test trial. Results supported the position that a stimulus associated with the onset of an acquisition session, but not with the extinction session, evokes greater response strength on a spontaneous recovery test trial than do other stimuli in the acquisition session. This holds true even if responses to this stimulus are never reinforced. In addition, it was found that, unlike a previous study that invoked five daily acquisition sessions, pigeons did not demonstrate increased responding to a novel stimulus.
\end{abstract}

Traditional explanations of spontaneous recovery include inhibition theories (e.g., Hull's, 1952, Ir) and interference theories (e.g., Guthrie, 1952). Another explanation was developed by Skinner (1950), who stressed that events outside the procedure can generate spontaneous recovery, particularly if stimuli immediately prior to the conditioning procedure are present before both the acquisition session and the spontaneous recovery test trial. Recognizing the importance of cues both outside and within a procedure, Estes (1955) suggested that a response becomes conditioned to a population of stimulus elements during acquisition. The availability of each of these elements fluctuates randomly during extinction. As a result, some elements that were never sampled during extinction are reintroduced later during the test trial, resulting in spontaneous recovery.

An interpretation that further develops Skinner's and Estes' explanations is supported by a series of more recent studies (Burstein \& Mackenzie, 1974; Burstein \& Moeser, 1971; McMillan \& Burstein, 1976; Welker \& McAuley, 1978). This interpretation states that, in general, any operation that emphasizes the similarity between the onset of the acquisition session and the spontaneous recovery test trial, while simultaneously stressing the dissimilarity between the extinction sessions and this test trial, will result in the characteristic increase in the response strength associated with spontaneous re-

This research was supported in part by National Science Foundation Grant BNS 79-08839 and was based on a BA honors thesis by the first author conducted under the direction of the second. Requests for reprints should be addressed to Mark D. Holder, Department of Psychology, University of California, Berkeley, California 94720. covery. For example, Burstein and Mackenzie (1974) began each of five acquisition sessions with different colored keylights. By associating a novel color with the onset of each acquisition session, but not with extinction, a sixth novel stimulus presented on the test trial evoked an increase in responding. This increase in responding was not found in a control group that was presented with only one novel stimulus on the test trial. From these findings, Burstein and Mackenzie concluded that the pigeons presented with a total of six novel stimuli had developed a "concept of novelty." Additional support for an interpretation that emphasizes the importance of the discriminability between the acquisition and extinction sessions (from now on called the discrimination interpretation) was found by McMillan and Burstein (1976). They demonstrated that a distinctive stimulus associated with the onset of five acquisition sessions was sufficient to increase response strength on the test trial even when the rest period was eliminated. The discrimination interpretation views the rest period as only one of an almost infinite number of possible procedures that generate spontaneous recovery by stressing the similarity between the test trial and the acquisition sessions while maintaining the dissimilarity between the test trial and the extinction sessions.

The present study was designed to further test the discrimination interpretation of spontaneous recovery in three ways. First, it was designed to determine whether a single presentation of a distinctive stimulus is sufficient to increase responding on the spontaneous recovery test trial. Second it was intended to determine whether a stimulus never paired with reinforcement, but associated with the onset of acquisition, could generate the response increment 
associated with spontaneous recovery. Third, it was designed to further explore the development of the "concept of novelty." Although the Burstein and Mackenzie (1974) study employed five presentations of a novel stimulus in order to establish increased responding to a novel stimulus, there was some evidence that one presentation might be sufficient. The present study, therefore, determined whether a novel stimulus would evoke increased responding during the test trial after a different novel stimulus, associated with the onset of the acquisition session, was presented only once.

\section{METHOD}

\section{Subjects}

Sixty-three experimentally naive White King pigeons, male and female, were housed in cages in a room illuminated in accordance with a 12/12-h light/dark equal cycle. The subjects were given free access to water, maintained at $80 \%$ of their free feeding weights, and randomly assigned to one of nine groups.

\section{Apparatus}

A Grason-Stadler operant-conditioning station (E1 100PE) was used. White noise was delivered into the operant chamber from a Grason-Stadler noise generator (E829E) to eliminate the effects of extraneous noise. A Grason-Stadler multiple stimulus projector (E4580) was modified to present the necessary stimuli. Three colors were presented on the keylight: red (Wratten Filter No. 821), green (Wratten Filter No. 856), and purple (Wratten Filter No. 846).

\section{Procedure}

Each pigeon was magazine and keypeck trained to a white keylight. The houselight was then removed, and each subject was given one acquisition session consisting of 20 trials of $15 \mathrm{sec}$ each, during which either a green or a red discriminative stimulus was on. The keylight was green on at least 19 of the 20 trials and was, therefore, labeled the standard stimulus. A red stimulus was presented on the first trial, the 15 th trial, or not at all during the acquisition session. Because the red discriminative stimulus was presented, at most, only once during acquisition, it was labeled the distinctive stimulus. All responses to the green stimulus were reinforced during acquisition with $3-\mathrm{sec}$ access to standard Purina pigeon pellets, as were responses by Groups 2, 3,4 , and 8 to the distinctive red stimulus. Responses by Groups 5-8 to the distinctive red stimulus were not reinforced. All discriminative stimulus trials were separated by a 15 -sec intertrial interval during which the key was not illuminated, the houselight was off, and no reinforcement was available. On the next day, all pigeons underwent extinction to the standard green stimulus until they reached the criterion of five consecutive trials without a response and then were returned to their home cages. After $30 \mathrm{~min}$, the pigeons were returned to the conditioning chamber for the 15-sec spontaneous recovery test trial.

As can be seen from Figure 1, Groups 2 through 9 differed in the three following ways: (1) They differed as to whether a distinctive red stimulus was presented on the first trial of acquisition or embedded on the fifteenth trial ( $F=$ first; $E=$ embedded). The embedded condition was employed to test whether any increase in responding to the distinctive stimulus presented on the first trial could be attributed to the fact that it had never been extinguished, rather than to its acting as a discriminative stimulus by being associated with the onset of acquisition. (2) They differed as to whether or not responses to the distinctive red stimulus during acquisition were reinforced $(R=$ reinforced; $U=$ unrein-

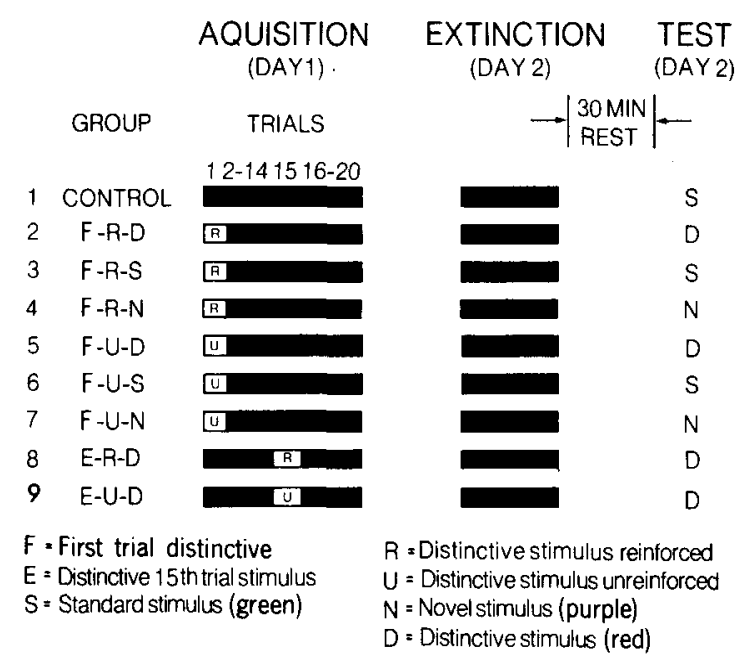

Flgure 1. The experimental design.

forced). The reinforcement condition was employed to determine whether the Burstein and Moeser (1971) study could be replicated using only 1 day of acquisition. The unreinforced condition was employed to determine whether reinforcement was necessary for a distinctive stimulus to increase responding during the spontaneous recovery test trial. (3) They differed as to whether the test trial consisted of the distinctive red stimulus, the standard green stimulus, or a completely novel purple stimulus $(D=$ distinctive; $\mathbf{S}=$ standard; $\mathbf{N}=$ novel). Presentation of the novel stimulus served as a control to determine whether any increase in responding to the distinctive stimulus, as compared with the standard stimulus, was a result of the position and discriminatory properties of the distinctive stimulus or whether any novel stimulus would increase responding, as in the Burstein and Mackenzie (1974) study.

In addition to these eight groups, Group 1, a control group, was presented with the standard green stimulus on all acquisition, extinction, and test trials. In other words, Group 1 received the standard spontaneous recovery procedure.

\section{RESULTS}

Three separate measures showed that prior to the test trial there were no differences among the nine groups. An analysis of variance showed that there were no significant differences between groups in response frequency over the last five trials in the acquisition session $[F(8,54)<1$; mean number of responses per trial $=4.1, S D=1.1]$. There were also no significant differences during extinction in response frequency on the first extinction trial $[F(8,54)$ $<1]$ or in the number of trials to the criterion of five successive trials without a response $[F(8,54)<1]$.

The measure of spontaneous recovery was the response frequency on the first test trial (the same measure employed by Burstein \& Mackenzie, 1974; Burstein \& Moeser, 1971; and McMillan \& Burstein, 1976). An analysis of variance based on this measure was significant $[F(8,54)=6.34, p<.001]$. Fisher's (1949) least significant difference procedure was performed in an attempt to identify the sources of the 
overall significance. No comparisons between Groups $1,3,4,6,7,8$, and 9 were significant (LSD $=3.842, p<.05$ ) (the largest difference between these sample means was 2.25 ).

During the test trial, Groups 2 and 5, the only two groups that received the distinctive stimulus on both the first trial of the acquisition session and on the test trial, responded at a significantly greater rate than the other seven groups $(\mathrm{LSD}=3.842, \mathrm{p}<.05)$. Furthermore, not only did the distinctive similarity between the onset of acquisition and the test trial increase responding, but reinforcement of the distinctive stimulus during acquisition also increased responding. This finding is shown by the result that Group 2, which had the distinctive stimulus reinforced, responded significantly more than Group 5, the nonreinforced distinctive stimulus group (LSD = $3.842, \mathrm{p}<.05$ ).

As can be seen from Table 1, in general, as the mean response rate for each group increases, so does the standard deviation. Because of this correlation, the data from the main groups of interest (Groups 1, 2 , and 5) were transformed using square roots. Findings based on the analysis of the transformed data were consistent with the main findings. An ANOVA of the transformed data showed a significant effect between the control group, Group 2, and Group $5[F(2,17)=4.57, p<.05]$. (The withingroups df has been reduced by 1 based on Box and Cox's, 1964, recommendations for when the data have been used to choose the transformation.) A trend analysis was then performed to determine whether the transformed response rate of Group 2 was greater than Group 5 and, in turn, if the response rate of Group 5 was greater than the control group's. A significant linear trend was found $[F(1,53)$ $=6.85, \mathrm{p}<.05]$ but no quadratic trend $[\mathrm{F}(1,53)<1$, $\mathrm{p}>.05]$. As was the case when the raw response rates were analyzed, the linear trend of the transformed data indicates that the response rates increased for the groups that received a distinctive stimulus at the onset of the acquisition session and during the test trial, and this increase was greatest when the distinctive stimulus was reinforced.

Table 1

Test Trial Responses by Groups

\begin{tabular}{crc}
\hline Group & Mean & SD \\
\hline 1 (Control) & 3.43 & 3.50 \\
2 (F-R-D) & 12.00 & 7.64 \\
3 (F-R $S)$ & 3.29 & 2.36 \\
4 (F-R-N) & 1.57 & 1.90 \\
5 (F-U-D) & 8.00 & 4.86 \\
6 (F-U-S) & 3.71 & 2.14 \\
7 (F-U-N) & 1.57 & 1.71 \\
8 (E-R-D) & 3.86 & 2.70 \\
9 (E-U-D) & 1.57 & 1.81 \\
\hline
\end{tabular}

\section{DISCUSSION}

In general, it seems that any operation that emphasizes the similarity between the onset of the acquisition session and the spontaneous recovery test trial while simultaneously stressing the dissimilarity between the extinction trials and these test trials will result in the characteristic recovery in response strength associated with spontaneous recovery. The results of this study show that Group 5 (F-U-D) responded significantly more to the test trial than either Group 1 (control) or Group 9 (E-U-D). These results show that a distinctive stimulus associated with the onset of the acquisition session can generate the increase in response strength associated with spontaneous recovery, even if this stimulus is not reinforced.

The finding that Groups 2 (F-R-D) and 5 (F-U-D) had the highest response rates on the test trial cannot be accounted for by any explanation appealing to secondary conditioning, stimulus generalization, or the fact that in Group 2 the distinctive stimulus was never extinguished. If these factors were responsible for the increased responding on the test trial, then Groups 8 (E-R-D) and 9 (E-U-D) should have also displayed this response increment because, except for when it was presented, the distinctive stimulus in these two groups was treated in exactly the same way as in Groups 2 and 5.

The present study confirms in part the findings and interpretations of the previous studies (Burstein \& Mackenzie, 1974; Burstein \& Moeser, 1971). In the Burstein and Moeser study, a greater increase in response strength on the test trial was found if the distinctive stimulus was associated with the onset of each of the five acquisition sessions of 30 trials than if the distinctive stimulus was presented as embedded on the 20th trial of each acquisition session. The present study, therefore, replicates the finding that the same stimulus generates different levels of responding depending on its position in the acquisition session. This is reflected by the significantly greater responding of Group 2 (F-R-D) as compared with Group 8 (E-R-D). Therefore, the present study indicates that the Burstein and Moeser (1971) study can be replicated by employing only one presentation of the distinctive stimulus instead of five.

This increase in response strength after only 1 day of acquisition was suggested by the Burstein and Mackenzie (1974) study, which found significantly greater responding on a test trial, as compared with a control group, to each of the five distinctive stimuli presented only once during acquisition. Each of the five distinctive stimuli was associated with the onset of a different acquisition session. Unlike the Burstein and Mackenzie study, however, the present study found that rate of responding to the novel stimulus 
in Groups 4 (F-R-N) and 7 (F-U-N) was not significantly greater than the control group. This finding suggests that a single presentation of a novel discriminative stimulus on the first trial of an acquisition session is not sufficient for the pigeon to associate novelty with the onset of acquisition.

Also of interest is the finding that responding to the novel stimulus in Groups 4 (F-R-N) and 7 (F-U-D) was not virtually totally inhibited as it was in the Burstein and Moeser (1971) study. Groups 4 and 7 of the present study differ from the Burstein and Moeser study because complete inhibition was obtained in the latter only when no other distinctive stimulus was presented. In the present study, a distinctive stimulus was presented during acquisition and a different distinctive stimulus was presented on the test trial of Groups 4 and 7 . This study suggests that even one additional distinctive stimulus presented during the acquisition session partially removes the inhibitory effect but is not sufficient to establish a novel stimulus as a significant cue for responding on the test. Two possible explanations of this finding are that the novel stimulus was partially, but incompletely, established as a response cue or that the repeated presentation of a novel stimulus in the absence of an aversive event reduces the inhibiting properties of another novel stimulus presented on the test trial because of habituation.

Although the Burstein and Moeser (1971) and the McMillan and Burstein (1976) studies found significant differences between the control group's response rates and the essentially totally inhibited response rates of groups tested with a novel stimulus, no difference was found between Group 1 (control) and Group 4 (F-R-N) of the present study. One possible explanation of this finding is that the difference was reduced in the present study because each group received one acquisition session and one extinction session, whereas the groups in the earlier studies received five acquisition and one extinction session. By receiving the same number of extinction and acquisition sessions, the effects of extinction may have been greater in the present study.

Although both groups that were tested with a distinctive stimulus associated with the onset of the acquisition session [Groups 2 (F-R-D) and 5 (F-U-D)] responded significantly more to the test trial than all other groups, Group 2 responded significantly more than Group 5. Since the only difference between Groups 2 and 5 was whether responses during the presentation of the distinctive stimulus during the acquisition session were reinforced, reinforcement clearly affects the capacity of a stimulus to evoke responding during a spontaneous recovery test trial. As noted, a nonreinforced distinctive stimulus can evoke greater responding than a control group on a spontaneous recovery test trial. If paired with reinforcement, however, the capacity of the stimulus to increase response strength is even greater. This difference between a reinforced and a nonreinforced stimulus' capacity to increase response strength suggests an additive model in which this capacity is determined by whether or not the stimulus is paired with reinforcement, the serial position of the stimulus, and whether or not it is distinctive.

The results of this study support the position that the traditional operation employing a rest period to evoke the characteristic recovery of response strength associated with the phenomenon of spontaneous recovery is only one of many possible operations that can produce the response increases associated with spontaneous recovery. This increase can be attributed to the similarity of the onset of the acquisition sessions to the test trial and to the discriminability between the extinction session and the test trial. Further research into the precise stimulus conditions that control the phenomenon of spontaneous recovery will determine whether the proposed additive model is useful and, if so, what other factors influence the capacity of a stimulus to evoke increased responding on the test trial.

\section{REFERENCES}

Box, G. E. P., \& Cox, D. R. An analysis of transformation. Journal of the Royal Statistical Society, 1964, 26, 211-243.

BURSTEIN, K. R. Spontaneous recovery: A (Hullian) noninhibition interpretation. Psychonomic Science, 1967, 7, 389-390.

Burstein, K. R., \& Mackenzie, L. Response strength as a function of stimulus novelty: Concept formation in the pigeon. Journal of Comparative and Physiological Psychology, 1974, 86, 142-145.

Burstein, K. R., \& Moesen, S. The informational value of a distinctive stimulus associated with the initiation of acquisition trials. Learning and Motivation, 1971, 2, 228-234.

Estes, W. K. Statistical theory of spontaneous recovery and regression. Psychological Review, 1955, 62, 145-154.

Fisher, R. A. The design of experiments. Edinburgh: Oliver \& Boyd, 1949.

GuThrie, E. R. The psychology of learning (Rev, ed.). New York: Harper, 1952.

HulL, C. L. A behavior system. New Haven: Yale University Press, 1952.

McMillan, R. D., \& Burstein, K. R. "Spontaneous recovery" following the elimination of the rest period. Bulletin of the Psychonomic Society, 1976, 8, 169-170.

Skinnen, B. F. Are theories of learning necessary? Psychological Review, 1950, 57, 193-216.

WelKer, R. L., \& McAuley, K. Reductions in resistance to extinction and spontaneous recovery as a function of changes in transportational and contextual stimuli. Animal Learning \& Behavior, 1978, 6, 451-457.

(Manuscript received September 4, 1980; revision accepted for publication June 16, 1981.) 